\title{
Downstream flow and upstream movement determine the value of a stream reach for potadromous fish populations
}

\author{
Yasmine Samia \\ Department of Mathematics and Statistics \\ University of Ottawa \\ Ottawa, ON, K1N6N5, Canada \\ Frithjof Lutscher \\ Department of Mathematics and Statistics, and Department of Biology \\ University of Ottawa \\ Ottawa, ON, K1N6N5, Canada
}

Corresponding author:

Frithjof Lutscher. email: flutsche@uottawa.ca. fax: 1-613-562-5776 


\begin{abstract}
Given that human activities often have negative impacts on biological populations, a common question is to find the location of greatest positive or least negative impact. Local habitat suitability is frequently used to evaluate viability of fish populations in river networks. Upper stream reaches are often undervalued, in particular when they are not navigable or do not contain commercially interesting fish. Since water flow transports certain local conditions downstream and individuals navigate river networks upstream and downstream, impacts of local perturbations can manifest elsewhere in the system, and overall effects of disturbances should be assessed on a network level. We study a model for a potadromous fish population in a system of connected stream reaches. We consider different geometries to evaluate how downstream transport and individual movement interact to determine the location of greatest and least impact of a single or two concurrent disturbances. Our results show how upper stream reaches can be highly significant for population persistence if downstream transport of abiotic conditions or upstream movement of individuals is strong.
\end{abstract}




\section{Introduction}

Human activities and land-use changes can cause local alterations to river reaches, often leading to deteriorating habitat conditions for fish. Water flow and fish movement carry the effects of local alterations to other locations in the watershed. Successful conservation efforts for freshwater fish therefore require an understanding of systemwide effects of these local changes (Stanfield et al., 2014). The branching structure of a river network can be represented as an interconnected set of habitat patches with several highly specific features that distinguish it from a terrestrial network of connected habitat patches and potentially drive population dynamics (Lowe, 2006). The dendritic geometry of branching river reaches and the downstream transport of physical conditions by water are two of these features that need to be considered when evaluating the effects of local alterations on global ecosystem properties (Fagan, 2002; Goldberg et al., 2010; Padgham and Webb, 2010). Our work aims to illuminate some of the complex interactions between abiotic conditions and population dynamic responses to local perturbations in river networks.

Different reaches in river networks have different functions that contribute to the overall well-being of the river system. While the main river channel and downstream reaches typically sustain a larger variety of species (Nilsson et al., 1994), headwaters regulate floods and sedimentation, support downstream food chains and contribute greatly to river biodiversity (Meyer et al., 2007; Bishop et al., 2008; Thorp et al., 2006; Finn et al., 2011). However, these upper reaches of a watershed are often overlooked when management efforts mainly focus on exploitable and navigable parts of the river (Stanfield et al., 2014). We regularly become aware of the strong connection between upstream disturbances and downstream effects when disastrous spills occur upstream and wipe out life downstream; for example recently from an old gold mine along the Animas river in Colorado (Moreno and Billeaud, 2015). But not all effects are so drastic or immediate. A recent workshop discussed the question of which and how many headwater drainage features could be sacrificed to development and agricultural use before fish assemblages downstream would be seriously affected (Stanfield et al., 2014).

The enormous consequences of disturbances make empirical work hazardous, limited or impossible. Mathematical models can help understand principles and mechanisms of population persistence in these networks. While there are many hydrological models describing water flow, they often do not include ecological dynamics, but see Jin et al. (2014). Ecological dynamics in river networks are frequently modeled with deterministic or stochastic patch- or metapopulation models (Charles et al., 1998b,a, 2000; Fagan, 2002; Goldberg et al., 2010; Labonne et al., 2008; Padgham and Webb, 
2010; Samia et al., 2015). These models usually consider movement of individuals between river reaches but not transport of local disturbances to downstream locations through river flow.

Downstream transport of chemical pollution was addressed explicitly in a series of detailed tactical ecotoxicological models by Chaumot et al. (2002, 2003a,b) in the context of cadmium effects on brown trout in a complete binary network of 15 reaches. Toxicant effects differed with the spatial pattern of exposure; in particular, the effect of pollution at the river mouth was much higher than in upstream reaches. This effect of space could be understood as a function of the proportion of adults exposed to pollution (Chaumot et al., 2003b) and interacts with migratory patterns during spawning (Chaumot et al., 2003a).

In this work, we develop and analyze a more general, strategic model to examine how the locations of a single or two separate local disturbances in a river network affect the fate of a hypothetical fish population. We consider a potadromous population, i.e. fish that spend their entire life cycle in freshwater. In our framework, a transportable disturbance can be chemical pollution but also other abiotic aspects, such as temperature or turbidity, that can affect reproductive rates and can be transported downstream by river flow. The model is based on our earlier work on the effect of movement barriers (e.g. dams or culverts) on the population growth rate in different dendritic geometries (Samia et al., 2015). One of our results there was that studying two, in some sense 'extreme', geometries sufficed to understand the behavior of the system in more natural, randomly generated networks. These two are the linear and the complete binary arrangement of river reaches. In the linear case, all reaches form a single linear chain, reminiscent of the lower Nile River when viewed from space. In the complete binary case, each reach is fed by exactly two immediate upstream reaches, comparable more to the vast network of the Amazon river. Our results here are based on these two extreme geometries. We introduce a disturbance in any reach in the network and evaluate how it changes the asymptotic population growth rate of a resident fish population. This growth rate is a crucial quantity in demography in that it indicates whether the population will persist or go extinct (Caswell, 2001). We study how this effect depends on the ease with which the disturbance is transported downstream and on the movement pattern of the fish. From our analysis, we formulate general principles for how these two mechanisms affect the location(s) of the 'most vulnerable reach(es)'; i.e., the location(s) where the population growth rate responds most strongly to the disturbance. By considering the joint effect of two disturbances, we also contribute to the study of cumulative effects of external drivers on population dynamics. Our results demonstrate the potential importance of a single reach on the fate of a population and can be applied 
to conservation efforts and land-use planning.

\section{The Model}

Our model tracks the density of a potadromous fish species in a dendritic river network from one year to the next. Each habitable reach in the network is considered a (homogeneous) patch of primary habitat and is represented as a node in the spatial graph. We exclude from the model the uppermost reaches of a river network where the fish species cannot survive, for example because they are too shallow or have too low volume.

An edge connecting nodes indicates a confluence of two reaches; see Figure 1. We denote by $N_{i, t}$ the density of the species in reach $i$ in year $t$. Between subsequent years, individuals first survive with probability $s_{i}$ and then produce, on average, $r_{i}$ offspring. For simplicity, there is no stage structure; i.e., offspring become mature adults within one year. An individual moves from reach $j$ to reach $i$ with probability $c_{i, j}$. Then the densities in a network of $n$ reaches evolve from year to year according to the equation (Samia et al., 2015)

$$
N_{i, t+1}=\sum_{j}^{n} c_{i, j}\left(r_{j}+s_{j}\right) N_{j, t} .
$$

As written, the model assumes that mature individuals first reproduce and then potentially die. Alternatively, the term $\left(r_{j}+s_{j}\right)$ can be seen as the expected total number of adults in the next generation generated from one adult in the current generation; acknowledging that this number contains the original adult itself if it survived.

This linear model is adequate to determine persistence conditions for the population as long as there are no Allee effects (Courchamp et al., 2008). The linear model also completely predicts the qualitative behavior of a corresponding nonlinear model, as long as the nonlinearities are non-decreasing and concave down functions (Samia, 2016).

Model equation (1) can be formulated as a linear matrix model for state vector $\mathbf{N}(t)=\left(N_{1, t}, \ldots, N_{n, t}\right)^{T}$ as

$$
\mathbf{N}(t+1)=B \mathbf{N}(t),
$$

where the projection matrix $B$ is the product of the migration matrix $C=\left(c_{i, j}\right)$ and the diagonal matrices for production and survival; i.e.,

$$
B=C[R+S] \quad \text { with } \quad R=\operatorname{diag}\left(r_{j}\right) \quad \text { and } \quad S=\operatorname{diag}\left(s_{j}\right)
$$




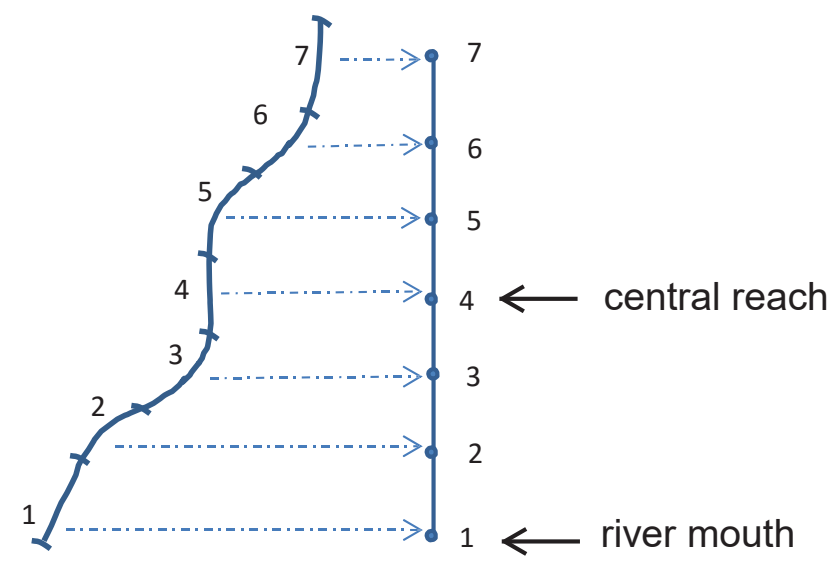

(a)

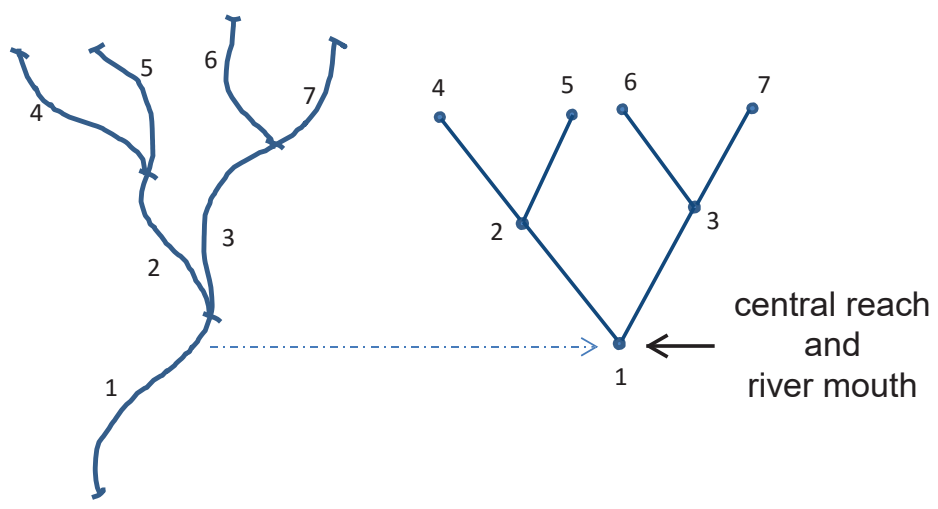

(b)

Figure 1: Representation of (a) a linear river network of 7 reaches (and 7 levels) and (b) a complete binary network of 7 reaches (on three levels) as spatial graphs. River reaches are represented by nodes and connections by edges. The "central reach" is the center of the tree graph in the graph-theoretic sense. It divides the graph into two equivalent subgraphs. 
Matrix $C$ is a (sub-) stochastic matrix that describes individual movement. We assume that individuals move by at most one reach per year, and we denote by $d_{i, j}$ $\left(u_{i, j}\right)$ the probability to move downstream (upstream) from patch $j$ to patch $i$. We assume that there is no loss or mortality related to movement, except possibly at the mouth of the river network. In particular, the column sums of matrix $C$ equal unity, except possibly for the first column. For the mouth of the river, we consider two extreme scenarios. If the first column sum equals unity, we speak of a closed system. No individuals leave through the river mouth. If $c_{1,1}=0$, we speak of an open system. An individual that does not move upstream from the mouth leaves the system; for more details, see Samia et al. (2015). For example, the connectivity matrices corresponding to the networks in Figure 1 are given by

$$
C=\left(\begin{array}{cccc}
* & d_{1,2} & 0 & 0 \\
u_{2,1} & 1-d_{1,2}-u_{3,2} & d_{2,3} & 0 \\
0 & u_{3,2} & 1-d_{2,3}-u_{4,3} & d_{3,4} \\
0 & 0 & u_{4,3} & 1-d_{3,4}
\end{array}\right)
$$

for the linear network, and by

$$
C=\left(\begin{array}{ccccccc}
* & d_{1,2} & d_{1,3} & 0 & 0 & 0 & 0 \\
u_{2,1} & 1-d_{1,2}-u_{4,2}-u_{5,2} & 0 & d_{2,4} & d_{2,5} & 0 & 0 \\
u_{3,1} & 0 & 1-d_{1,3}-u_{6,3}-u_{7,3} & 0 & 0 & d_{3,6} & d_{3,7} \\
0 & u_{4,2} & 0 & 1-d_{2,4} & 0 & 0 & 0 \\
0 & u_{5,2} & 0 & 0 & 1-d_{2,5} & 0 & 0 \\
0 & 0 & u_{6,3} & 0 & 0 & 1-d_{3,6} & 0 \\
0 & 0 & u_{7,3} & 0 & 0 & 0 & 1-d_{3,7}
\end{array}\right)
$$

for the complete binary network, where

$$
*= \begin{cases}0 & \text { if the system is open } \\ 1-u_{2,1} & \text { if the system described by (4) is closed } \\ 1-u_{2,1}-u_{3,1} & \text { if the system described by (5) is closed }\end{cases}
$$

We now describe how local and upstream conditions affect downstream population vital rates. For simplicity, we consider the case that only offspring production $\left(r_{i}\right)$ and not adult survival $\left(s_{i}\right)$ are affected. We distinguish the contribution of three groups of influences on production within a reach: (i) instream conditions $\left(\gamma_{i}\right)$, such as benthic substrate or level of shading (local, non-transportable); (ii) landscape conditions $\left(q_{i}\right)$, such as natural, industrial or agricultural run-off (local, transportable); and (iii) upstream conditions $\left(h_{i}\right)$, such as nutrient loading or temperature, which indirectly account for influences of upstream landscape conditions (non-local, transportable). 
This distinction is particularly relevant in our modeling approach since it allows us to keep track of the effects of local and remote environmental factors and separate them into in-stream and external conditions. Accordingly, we write the local growth rate as

$$
r_{i}=\exp \left(\gamma_{i}+q_{i}+h_{i}\right)>0 .
$$

We consider instream conditions to be fixed at their respective reach, whereas landscape and upstream conditions can be transported to downstream reaches by water flow. Hence, considering only local, instream conditions - i.e., setting $q_{i}=h_{i}=0$ our model reduces to the one we had previously used to study the effect of movement barriers on population persistence (Samia et al., 2015). We choose $q_{i}=0$ to be the neutral value, so that $q_{i}>0$ indicates a positive effect of landscape conditions on fish growth and $q_{i}<0$ a negative effect such as contaminants entering the river. Our analysis below focuses on the latter scenario.

To calculate the effect of upstream conditions on local growth rates, we take a weighted average of transportable upstream conditions according to relative discharge and discounted for distance. Specifically, if reaches $j$ and $k$ are directly upstream of reach $i$ (see Figure 1), we set

$$
h_{i}=f_{i j}\left(q_{j}+h_{j}\right) \operatorname{disc}_{i j}+f_{i k}\left(q_{k}+h_{k}\right) \operatorname{disc}_{i k},
$$

where $f_{i j} \geq 0$ is the fraction of discharge from reach $j$ into reach $i$, given by

$$
f_{i j}=\frac{\text { discharge from } j}{\text { total discharge in } i}
$$

and $\operatorname{disc}_{i j} \geq 0$ is the discounting factor between reaches $i$ and $j$. We measure discharge at the end of each reach, so that the total discharge in a reach equals the sum of the discharges of the reaches immediately upstream plus the lateral input. Furthermore, we assume that the discounting factor decreases with distance, and set

$$
\operatorname{disc}_{i j}=e^{-\nu_{i} l_{i}}
$$

where $l_{i}$ is the length of reach $i$. If the buffering factor $\nu_{i}$ is high, then there is little or no downstream transport. The upstream influence on downstream reproduction is low. This situation occurs if run-off contaminants are quickly moved into hyporheic storage zones and broken down there. On the other hand, if $\nu_{i}$ is low, then there is strong downstream transport, and upstream conditions have a large influence on downstream fish production. 


\section{Methods and assumptions}

We measure how a local (negative) disturbance affects the fish population, depending on the strength of downstream transport, fish movement preference and habitat heterogeneity. In particular, we determine which reaches are the most vulnerable (or valuable) for the population by reporting how the dominant eigenvalue $(\lambda)$ of projection matrix $B$ changes with respect to location and strength of the disturbance. For large disturbances, we report absolute changes, while for small perturbations, we report sensitivity analysis (Caswell, 2001).

We formulated our model in sufficient generality so that it is applicable to many different real-world river systems, from the linearly shaped lower Nile to the highly branched Amazon. However, for our detailed investigations reported here, we choose several carefully selected model simplifications that allow us to theoretically explore common principles rather than specific systems. We choose two extreme types of networks, a linear network where each non-boundary reach has exactly one upstream and one downstream neighbor, and a complete binary network where each non-boundary reach has exactly two upstream and one downstream neighbors. Our previous work shows that the dominant eigenvalue of intermediate, randomly generated networks is bounded by these two extremes (Samia et al., 2015). We illustrate our results using a linear network with seven reaches. In this case, each reach represents a different level (i.e., distance from the mouth of the river). For a complete binary network, we use one with 127 reaches (corresponding to seven levels) and one with 7 reaches (corresponding to three levels). Our qualitative results do not change when more levels are involved.

We make the simplifying assumptions that all reaches are of the same length, that the amount of lateral influx is negligible compared to downstream discharge, that the buffering factor and survival rates are constant throughout the network and that all reaches within one level are identical with respect to local conditions. In other words, we assume that the tree graph is radial (Sarhad et al., 2014). Therefore the value of $\gamma_{i}$ depends only on the level of the reach. In the simplest case, we consider a homogeneous network, where all local conditions are equal. To represent heterogeneity, we consider a fixed ratio $\rho$ of local growth rates between levels,

$$
\rho=\frac{\gamma_{\text {level }(k+1)}}{\gamma_{\text {level } k}}
$$

In particular, the case $\rho>1$ models a species that does better upstream (e.g., coldwater preference), whereas setting $\rho<1$ models a species that reproduces better downstream. 
Movement probabilities consist of an overall probability $(p)$ to move away from the current reach and a movement bias. In the absence of movement bias, the probability to move downstream is $d_{i, j}=p / 2$ and the probability to move upstream is either also $u_{i, j}=p / 2$ (in the linear network) or $u_{i, j}=p / 4$ to each of the two upstream connecting reaches (in the complete binary network). With bias upstream, the downstream movement probability is $d_{i, j}=p / 4$, and the upstream probabilities are adjusted accordingly. With bias downstream, the numbers are reversed. The probability to move upstream from the highest level is zero. The probability of moving downstream from the mouth of the river depends on whether the network is closed or open (see previous section). We initialize discharge in the highest level of the network as a constant, identical for all top-level reaches, and then calculate discharge throughout the network.

Unless otherwise noted in the text or figure caption, we choose parameter values as listed in Table 1.

\begin{tabular}{c|c|c} 
parameter & meaning & value \\
\hline$s_{i}$ & survival probability & 0.5 \\
$\nu_{i}$ & buffer factor & 0.01 (low) \\
& & 1 (high) \\
$q_{i}$ & landscape conditions & 0 (neutral) \\
& & -5 (disturbance) \\
$l_{i}$ & reach length & 10 \\
$\gamma_{i}$ & instream conditions & 0.5 \\
$p$ & overall moving probability & 0.5
\end{tabular}

Table 1: Default parameter values used for numerical results.

\section{Results}

Even with the simplifying assumptions made in the previous section, there is still a large number of scenarios to explore. Between two network types (linear, binary), two mouth conditions (open, closed) and three local quality options $(\rho=1, \rho>1$ and $\rho<1$ ), there are twelve scenarios, and for each there are three mechanisms to check (downstream transport, movement bias and movement probability). We begin by introducing a single disturbance (strong or weak) at exactly one reach and evaluating the response in $\lambda$. Then we consider the interaction of two disturbances and ask what their cumulative effect is. The results of our numerical explorations are summarized in the following sections. 


\subsection{Single disturbance in a homogeneous network $(\rho=1)$}

We choose the linear, homogeneous, closed network as our baseline scenario. We illustrate the three main findings in this case and report how they depend on the shape and other properties of the network.

I. The importance of reaches shifts in the direction of movement bias. Figure 2(a) depicts the value of $\lambda$ in the linear network depending on the level (reach) at which the disturbance is located. The value of $\lambda$ in the undisturbed network is given where the curves intersect the $y$-axis. If a strong, negative disturbance is placed anywhere in the network, the population growth rate decreases. In the absence of movement bias and downstream transport (i.e., when buffering is strong), the largest decrease occurs when the disturbance is placed in the "central reach" of the network. By "central reach" we mean a mid-point with respect to the network topology; this differs between the linear and the complete binary network (see Figure 2). The solid line in Figure 2(a) shows that the effect in a linear network is strongest at this middle or central reach. We shall refer to the location with the strongest effect as the critical disturbance location or the most vulnerable reach. With upstream (downstream) bias, the critical location moves upstream (downstream); see the dashed and dash-dot lines, respectively, in Figure 2(a).

When the network is open, downstream reaches are inherently more risky for individuals because they might leave the network at the river mouth (Samia et al., 2015). Accordingly, the most vulnerable reaches shift upstream, but their relative order on the three curves in Figure 2(a) remains the same (plot not shown).

II. Downstream transport increases the importance of upstream reaches. The bars in Figure 2(b) indicate the sensitivity of $\lambda$ with respect to a small disturbance at the corresponding location (i.e., $d \lambda / d q_{i}$ ). In the absence of downstream transport (i.e. buffering is strong) and movement bias, $\lambda$ is (almost) equally sensitive to disturbances in all reaches (dark bars in Figure 2(b)). In contrast, $\lambda$ is much more sensitive to disturbances in upstream reaches when buffering is weak and the disturbance is transported downstream (light bars in Figure 2(b)). The critical disturbance location is then shifted upstream. In fact, the difference between strong and weak buffering can make the difference between population persistence $(\lambda>1)$ and population extinction $(\lambda<1)$. For example, when a strong enough negative disturbance is placed in an upstream reach (level 7) and buffering is weak, then the population may face extinction, even though it would be able to persist if a disturbance of the same strength had been placed further downstream or buffering was strong; see Figure 2(c). This qualitative result is exactly the same for an open network (plot not shown). 
Comparing the (solid) line for unbiased movement in Figure 2(a) and the constant sensitivity in the absence of transport in Figure 2(b), we note that sensitivity analysis for small disturbances may not reveal the system's response to large disturbances, even though the system is linear. The dark bars indicating sensitivity in the absence of downstream transport seem constant on this scale, so that one could conclude that all reaches are equally vulnerable to disturbance. However, the bars actually differ by very small amounts. In particular, the highest sensitivity of $\lambda$ to a negative disturbance is observed at reach 4 , while its lowest corresponding sensitivity to negative disturbances is at reaches 1 and 7 . When a large disturbance is applied, these differences are amplified, and the higher vulnerability in the center reach becomes visible in Figure 2(a).

III. Movement rate enhances but does not change the pattern. When the overall probability of movement $(p)$ is small, then change in $\lambda$ with respect to a strong disturbance is very small. When the movement probability is large, then the variation in $\lambda$ increases significantly when a strong negative disturbance is introduced. The critical disturbance location, however, is independent of $p$, see Figure 2(d). With a high movement rate, individuals will pass through the disturbed reach more frequently, and therefore the effect is more pronounced. For an open network, again, the pattern is exactly the same (plot not shown).

$I V$. A broadly branched network decreases the importance of a single upstream reach and increases the importance of the central reach. In a complete binary network, the central reach is located at the mouth of the river. At the same time, there are $2^{k}$ identical reaches on level $k$ so that the importance of an individual reach is naturally diminished. These insights are crucial to understand the location of the most vulnerable reach in a complete binary network.

In a closed network, this most vulnerable reach is still the central reach of the network, which is now the river mouth. Hence the critical location is the lowest level. Upstream (downstream) bias does not shift this location but rather diminishes (enhances) the overall negative effect of the disturbance on $\lambda$; see Figure 3(a).

Even with downstream transport, the most vulnerable reach remains the river mouth, but many reaches in higher levels are almost equally vulnerable; see Figure $3(\mathrm{~b})$. In contrast, when there is no downstream transport, the effect of a disturbance is significantly smaller for any level upstream of the mouth; see Figure 3(a).

The heightened importance of upstream reaches through downstream transport of disturbances is also visible in the sensitivity analysis in Figure 3(c). In the absence of transport, $\lambda$ is by far the most sensitive to disturbances in the first level (river mouth), but, with transport, disturbances in the second level are almost as important. If a binary network is open, then the central location is also the most dangerous since 


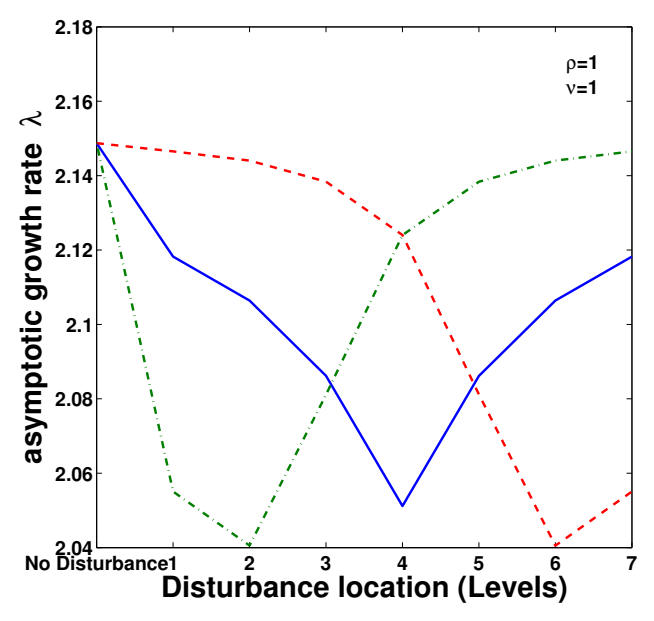

(a)

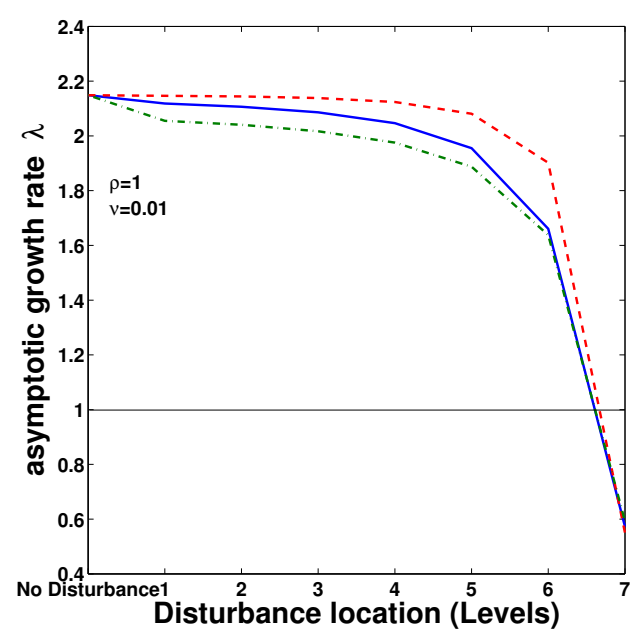

(c)

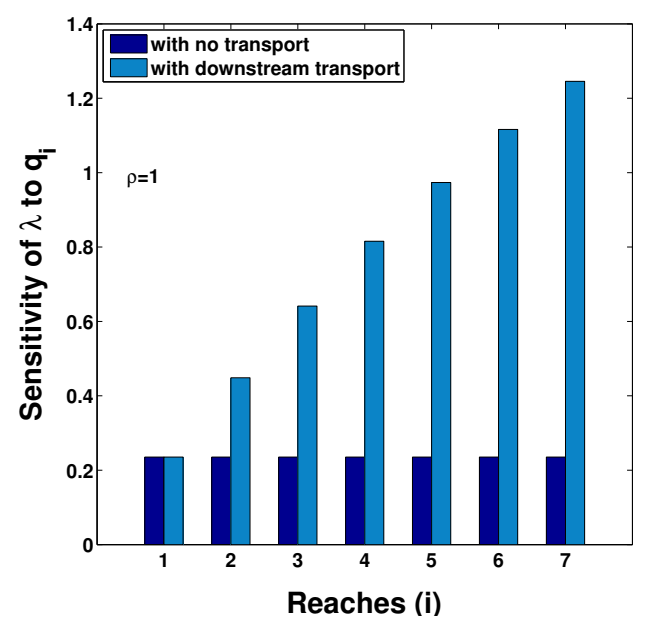

(b)

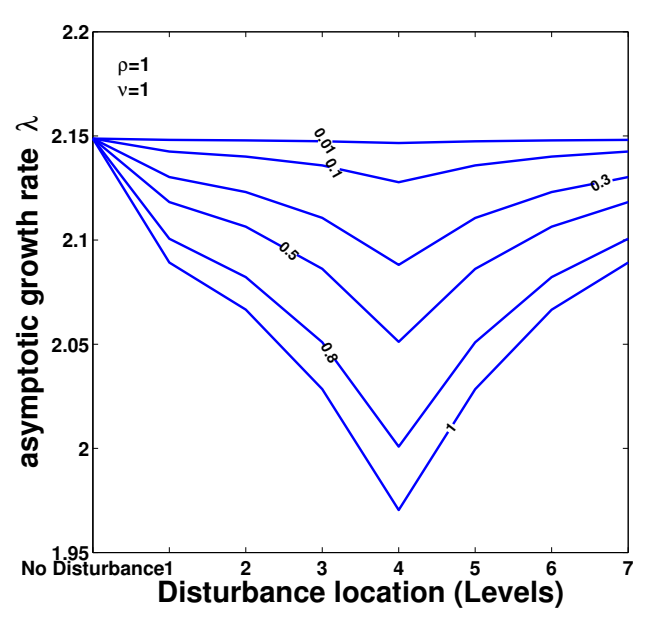

(d)

Figure 2: The effect of movement bias, downstream transport and movement probability on the population growth rate $\lambda$ in a linear, homogeneous closed network. (a) Population growth rate as a function of disturbance location for unbiased (solid), upstream biased (dashed) and downstream biased (dash-dot) movement in the absence of downstream transport. (b) Sensitivity of $\lambda$ with respect to disturbance location without (dark) and with (light) downstream transport. (c) Growth rate as a function of disturbance location with downstream transport; movement bias of individuals is indicated by line style as in (a). (d) Growth rate as a function of disturbance location for different values of overall movement probability. Parameters are as in Table 1 and in the annotations of the plots. 
individuals leave the network through the river mouth. Accordingly, an additional disturbance to the river mouth has no significant effect on the population growth rate. Therefore $\lambda$ is much less sensitive to the disturbance location than to movement bias, where upstream bias keeps individuals away from the river mouth and therefore increases $\lambda$ (plot not shown). A comparison of the sensitivity analysis also confirms that, in this case, downstream transport of conditions increases the importance of upstream reaches for population persistence, with the critical locations (all reaches in the same level) being around the mid-height of the network; see Figure 3(d).

To complete the description of our results, we mention that, in all cases, the overall movement rate $(p)$ has the effect of enhancing the existing pattern without changing it qualitatively.

\subsection{Single disturbance in a heterogeneous network}

The scenario that population growth rate is homogeneous in the network is not very realistic in practice. Habitat heterogeneity in river networks often occurs via gradients, say, as water temperature and nutrient load usually increase downstream whereas shading decreases (Vannote et al., 1980). Accordingly, we explore how the insights from the previous sections carry over to such gradient environments.

$V$. The importance of a reach (or the reaches in one level) increases when the population growth rate in these reaches increases. This insight is not at all surprising, but it is modulated and sometimes overwritten by the four previous findings above. We look at some of these aspects in more detail and begin with the scenario where the growth rate increases downstream.

\section{Growth rate increases downstream $(\rho<1)$}

When population growth rates are higher downstream, the importance of downstream locations is enhanced (if possible) and that of upstream locations is diminished. For closed networks, the effects are qualitatively similar to the case of a homogeneous network, just shifted downstream (plots not shown): the river mouth is typically the most vulnerable reach in the absence of downstream transport of the disturbance. With transport (i.e., weak buffering) upstream reaches become more important again. Upstream (downstream) movement bias gives smaller (larger) population growth rates. Sensitivity of $\lambda$ with respect to disturbances in upstream reaches increases considerably with downstream transport.

The situation is more interesting when the network is open because of two conflicting effects. The reach at the mouth of the river has the highest productivity but 


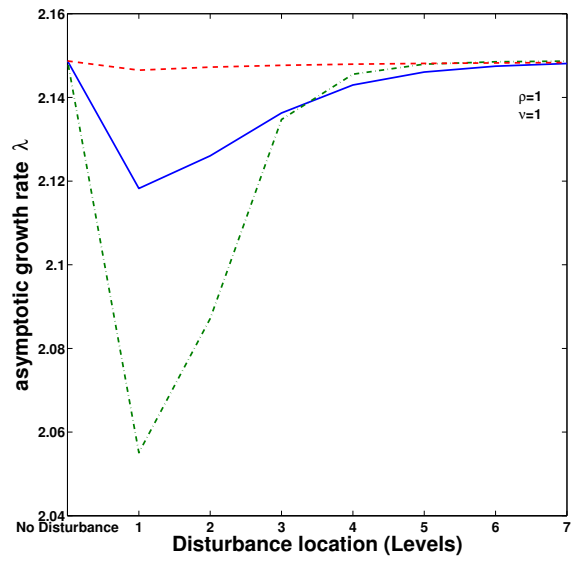

(a)

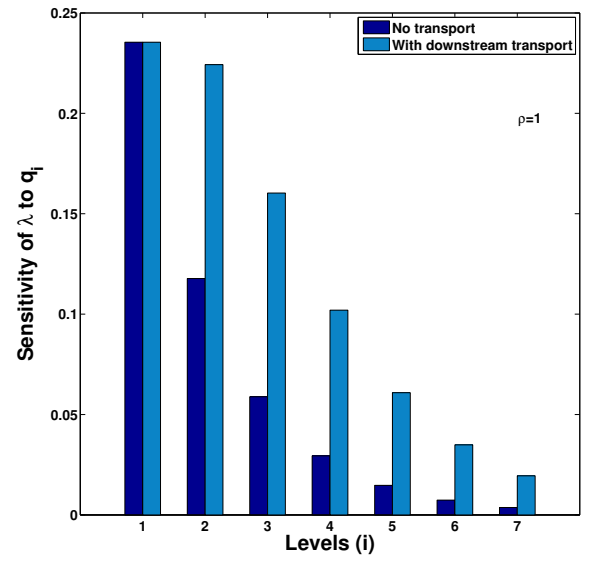

(c)

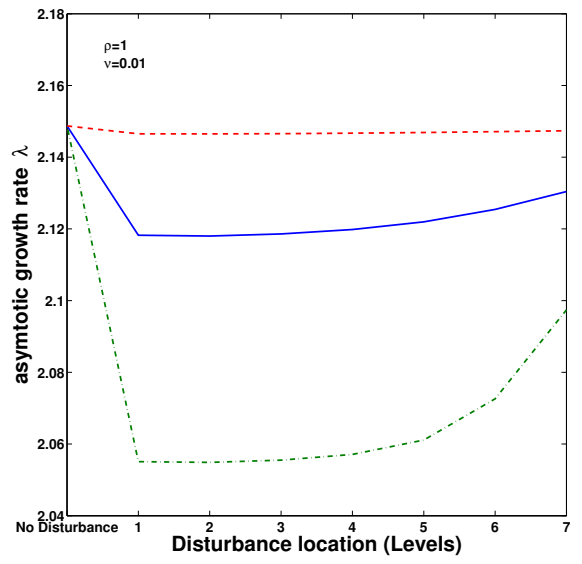

(b)

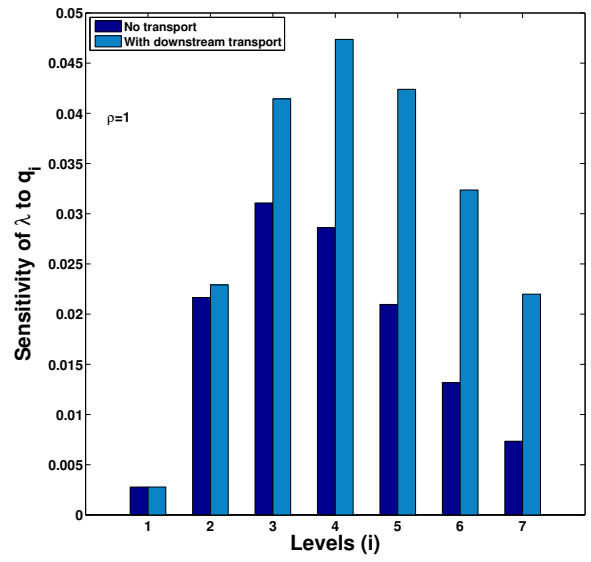

(d)

Figure 3: The effect of movement bias and downstream transport on the population growth rate $\lambda$ in a homogeneous complete binary network. (a) Population growth rate as a function of disturbance location for unbiased (solid), upstream biased (dashed) and downstream biased (dash-dot) movement, in the absence of downstream transport. (b) Same as (a) but with downstream transport. (c) Sensitivity of $\lambda$ with respect to disturbance location without (dark) and with (light) downstream transport in a closed network. (d) Same as (c) but in an open network. Parameters are as in Table 1 and in the annotations of the plots. 
at the same time carries the greatest risk of washout from the system. This risk of washout in fact decreases the importance of downstream reaches. Mid-level reaches become more important in the linear and binary networks. Figure 4(a) demonstrates how downstream transport enhances the importance of upstream reaches in the linear network; Figure 4(b) is the corresponding plot for the complete binary network.

As before, increasing the overall movement probability $(p)$ enhances the pattern. When there is no transport, increasing $p$ can also shift the critical location upstream; with strong transport, the critical location is and remains upstream.

\section{Growth rate increases upstream $(\rho>1)$}

When the population growth rate is higher upstream, the upstream reaches are inherently more important. Upstream bias in movement enhances this trend, as does downstream transport of disturbances. In a linear network, the critical disturbance location is moved upstream, as compared to the homogeneous case. This effect is clearly visible in the absolute values of $\lambda$ and in the sensitivities, and it is independent of whether the river mouth is open or closed. The larger the disturbance is, the lower $\lambda$ gets. Without transport, the critical location in the linear network is the second highest level, and the population can persist in all cases; see Figure 5(a). With transport, the critical location is the highest level, and the population is not viable when a large disturbance is located there $(\lambda<1)$; see Figure $5(\mathrm{~b})$. The sensitivity of $\lambda$ with respect to a disturbance in the highest level with transport is nearly twice that without transport; see Figure 5(c).

In the complete binary network, both open and closed, the importance of the river mouth as the central reach is diminished since growth rates are higher upstream. The population growth rate is most sensitive to disturbances in the second highest level when there is no transport, and in the highest level when there is transport; see Figure 5(d). We note, however, that the sensitivity values are very small. While the importance of the river mouth is diminished by the upstream increase in growth rate, the importance of any single upstream reach is diminished by the fact that there are $2^{k}$ such reaches on level $k$. Accordingly, the population growth rate appears almost constant with respect to disturbance location (plot not shown).

\subsection{Two disturbances and cumulative effects}

So far, we have considered how the population growth rate in a pristine river network is affected by the location of a single disturbance, large or small. But what happens when there is already a disturbance present in the network? Where would an additional disturbance have the largest effect, and what is the cumulative effect 


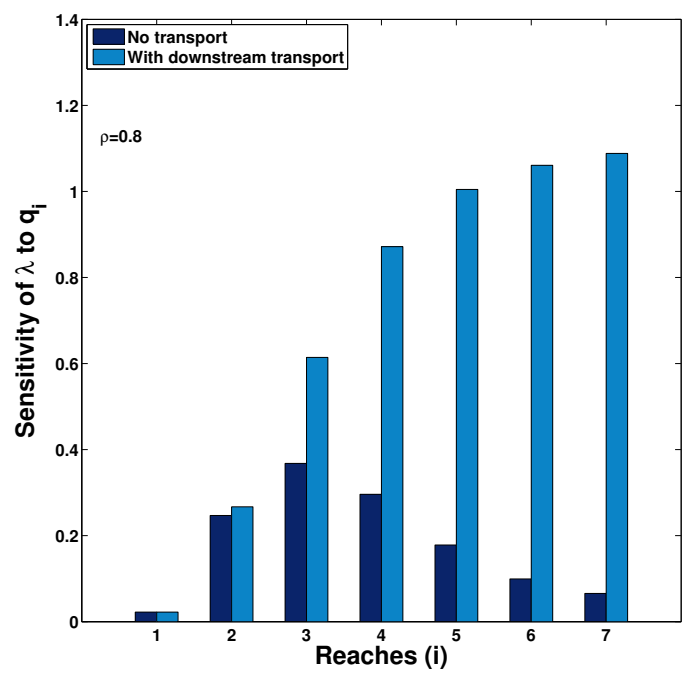

(a)

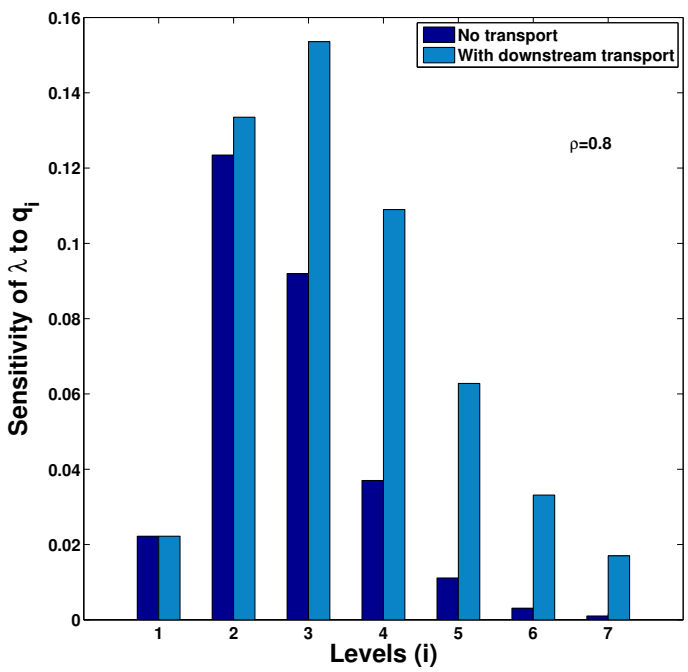

(b)

Figure 4: The effect of downstream transport when downstream locations provide better growth conditions. Sensitivity of $\lambda$ with respect to disturbance location without (dark) and with (light) downstream transport in (a) an open linear network and (b) in an open complete binary network. Growth rate increases downstream; i.e., $\rho<1$. Parameters are as in Table 1 and in the annotations of the plots. Additional plots of the (absolute) growth rate for this and many other scenarios are available in Appendix D of Samia (2016). 


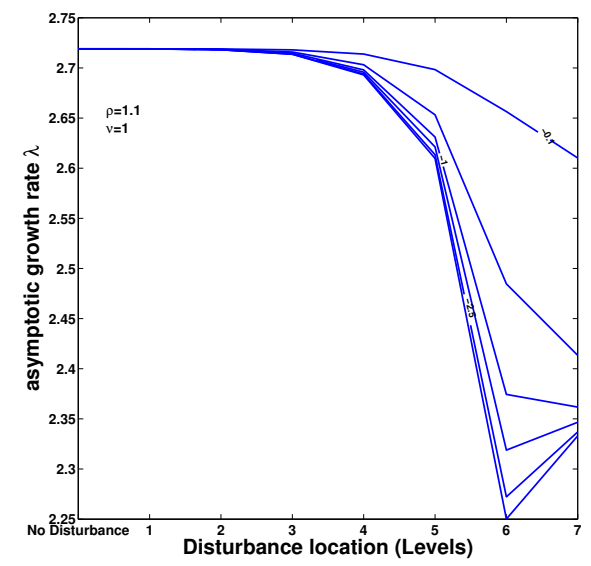

(a)

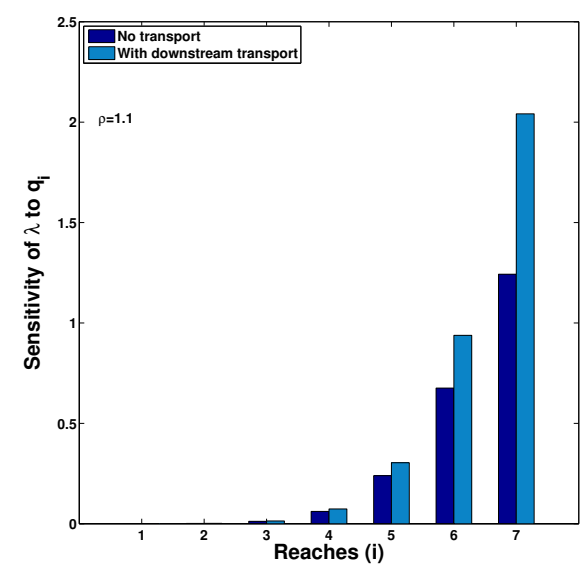

(c)

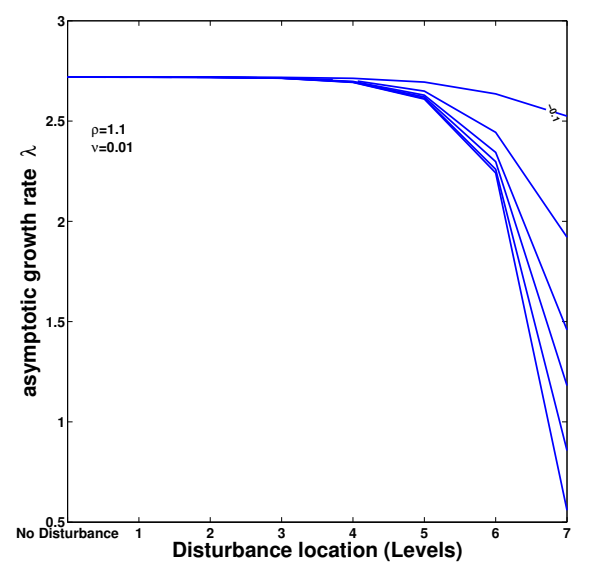

(b)

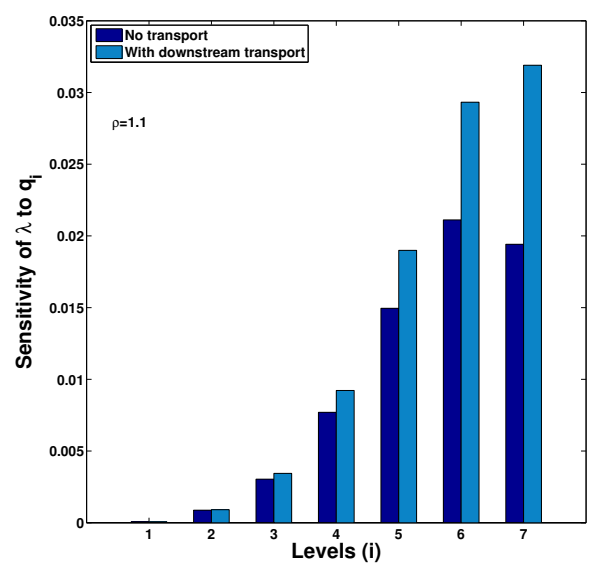

(d)

Figure 5: The effect of downstream transport when upstream locations provide better growth conditions. Population growth rate $\lambda$ in a linear network with different disturbance intensity ranging from $q=-0.1$ to $q=-5$ and no movement bias: (a) without downstream transport, (b) with downstream transport. The results hold for both, the closed and open linear network. Sensitivity of $\lambda$ with respect to disturbance location without (dark) and with (light) downstream transport in (c) an open linear network and (d) in an open complete binary network. Growth rate increases upstream, i.e. $\rho>1$. Parameters are as in Table 1 and in the annotations of the plots. 
of the two disturbances? We answer this question by sensitivity analysis; i.e., by calculating the change in $\lambda$ with respect to the location of a second disturbance, given the location of the existing disturbance.

Whereas there are only $n$ possible locations for a single disturbance in a network of $n$ patches, we now have to deal with $n^{2}$ possible pairs of locations for the two disturbances. For that reason, we illustrate our results only for linear and complete binary networks of seven reaches each. (Accordingly, the binary network will have only three levels). We checked our findings for larger networks, and found the same qualitative patterns. Corresponding plots for these larger networks can be found in the appendix of Samia (2016).

VIa. In the linear network, a secondary disturbance has the strongest effect if it is farthest from the primary disturbance. Upstream transport shifts this location upstream. Figure 6(a) shows the sensitivity of $\lambda$ in a linear network with respect to a small disturbance in reaches 1-7 for a given existing disturbance in reaches 17 as a 3D bar plot. In the absence of downstream transport and movement bias, the population growth rate is insensitive to a further small disturbance at the same site. The bars along the diagonal are essentially zero. The largest sensitivity arises when the distance between the additional disturbance and the already-disturbed site is the longest. When the existing disturbance is downstream (in reaches 1-3), the highest bars are at the source (reach 7), whereas when the existing disturbance is upstream (reaches 5-7), then the highest bars are at the river mouth (reach 1). Downstream transport shifts this pattern such that upstream locations are much more influential; only when the original disturbance is in the highest reaches do additional disturbances in the downstream locations have a significant effect on $\lambda$; see Figure 6(b). With upstream movement bias, the same effect arises. Downstream movement bias shifts the importance of locations downstream, but when conditions are transported downstream as well, the most influential locations are again upstream (plot not shown).

$V I b$. In the binary network, a secondary disturbance has the strongest effect if it is at the root or close to the root but on the opposite side of the primary disturbance. Again, upstream transport shifts this location upstream.

In a complete binary network (see Figure 1 for labeling of the reaches), the central reach is the most important. In the absence of downstream transport and movement bias, the sensitivity is smallest for disturbances occurring at the already-disturbed site. If the primary disturbance is at the mouth, then the strongest effect for a secondary disturbance is in the highest level. If the primary disturbance is far from the mouth, then the strongest effect for a secondary disturbance is the mouth. Only a disturbance in level 2 has a strongest secondary disturbance away from the mouth, 
namely at level 2 on the other side of the mouth; see Figure 6(c). With downstream transport, the most influential locations are the ones farthest away from the existing disturbance; i.e., in the highest level on the half of the network that is opposite (with respect to the river mouth) of the original disturbance; see Figure 6(d). For example, if the original disturbance is in reach 2, then the furthest reaches are 6 and 7 . Just as with the linear network, this pattern also arises with upstream bias and persists with upstream or downstream bias, as long as there is downstream transport. When there is downstream bias but no downstream transport, the river mouth regains its status of the most influential location (plot not shown).

Qualitatively, these findings remain true when the network is not homogeneous but rather contains the gradient structure for either $\rho>1$ or $\rho<1$. As before, upstream (downstream) levels become more important when upstream (downstream) conditions are better. However, downstream transport and upstream movement bias continue to make the upstream reaches the most vulnerable ones.

\section{Discussion}

Questions about how water safety and biodiversity are affected by river network alterations - e.g., through climate change, urban sprawl or agriculture - are of paramount importance. The complexity of river ecosystems, governed by the interactions of geomorphology, hydrology, connectivity and ecology, poses great challenges in the understanding of mechanisms and effects. Management of these systems frequently relies on hydrological models and local habitat suitability measures to evaluate the impact of alterations on a given species but tends to neglect ecological feedbacks (Anderson et al., 2006). Since water flow transports many physical and geochemical aspects from upper to lower reaches, questions about the importance of upper reaches and about cumulative downstream effects are particularly pressing (Stanfield et al., 2014). Our strategic analytical model contributes some mechanistic insights into these questions.

We studied how the asymptotic growth rate of a hypothetical potadromous fish species changes with respect to disturbances in one or two reaches in the network, especially how the change depends on transport of the disturbance. In this way, we identified the most vulnerable reaches for a negative disturbance. Clearly, local habitat suitability is important (see $V$ ), but movement bias (see $I$ ) and downstream transport (see $I I$ ) can move the critical location upstream of the best local habitat and increase the importance of upper reaches. Species with high movement rates show a more pronounced but qualitatively similar pattern as species with low movement rates (see $I I I)$. A single upstream reach is less influential in a highly branched 


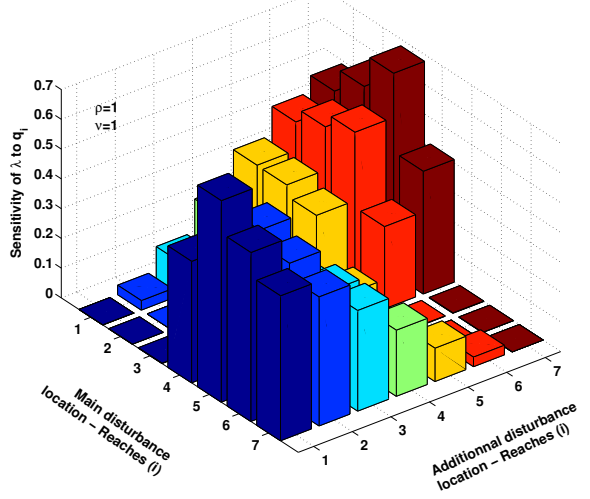

(a)

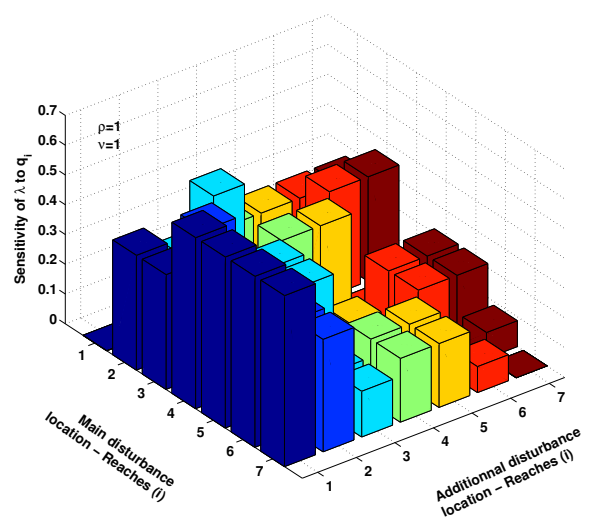

(c)

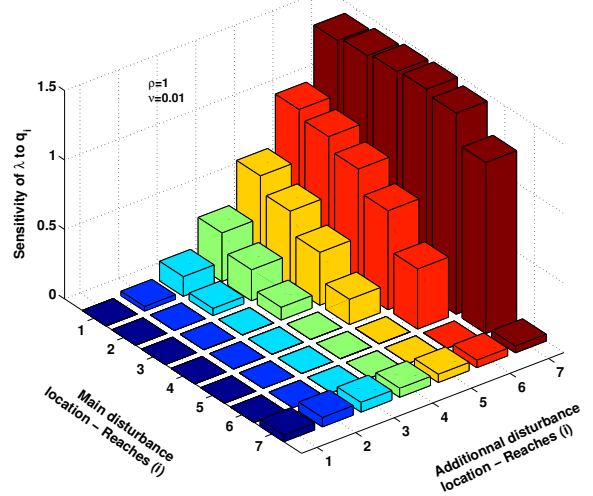

(b)

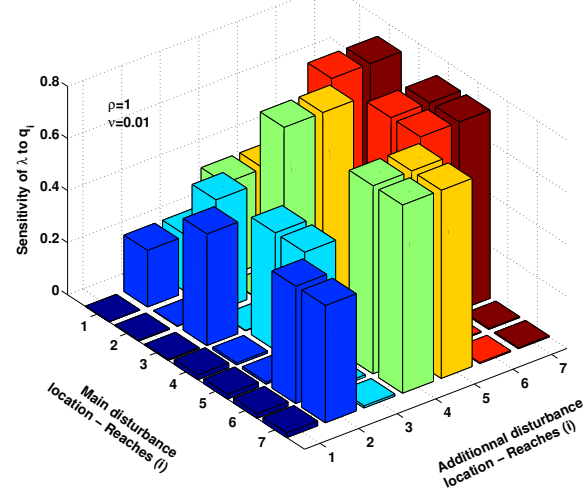

(d)

Figure 6: Cumulative effects for two disturbances in different geometries. Sensitivity of $\lambda$ to a secondary disturbance in a closed linear network (a) without transport and (b) with transport, as well as in a closed complete binary network (c) without and (d) with transport. Parameters are as in Table 1 and in the annotations of the plots. 
structure (see $I V)$, but can have significant importance for a secondary perturbation when there is significant downstream transport (see $V I b$ ).

Our study of population dynamics in a discrete dendritic network and discrete time complements recent studies with continuous dendritic networks in continuous time (Ramirez, 2012; Sarhad et al., 2014; Sarhad and Anderson, 2015). Those authors considered organisms that cannot actively swim against the current (e.g. plankton and stream insects). They considered how network size and topology affect the dominant eigenvalue, but did not consider disturbances. They found some qualitatively similar results, for example, regarding the importance of individuals staying away from lethal boundaries, and the usefulness of upstream refuges.

Our results highlight the importance of upstream reaches in a dendritic network when disturbance attributes are easily transported downstream. As the asymptotic growth rate is a global indicator for the entire network, our study complements mechanistic models on smaller scales that looked at response length for population distribution on a reach level (Anderson et al., 2005). Vice versa, this finding suggests that mitigation or restoration immediately downstream of a disturbance would have the greatest benefits. Such measures can include buffer zones (Correll, 1996) and other measures to shorten uptake length (Lutscher and McCauley, 2013).

Our results also point out that details of the movement behavior of fish in river networks can be crucial for population persistence and need to be accounted for when assessing potential effects of disturbances. This aspect of the study complements our previous work where we considered movement behavior around barriers, such as dams or culverts (Samia et al., 2015). Populations with upstream movement bias are more vulnerable to disturbances in upper reaches. For example, we would expect upstream bias in cold-water species so that upstream disturbances could be more detrimental for bull trout (Salvelinus confluentus), which require $<13^{\circ} \mathrm{C}$ temperatures, than for brook trout (Salvelinus fontinalis), which can tolerate up to $22^{\circ} \mathrm{C}$ temperatures. While it is clear that our model does not apply to fish species with completely different movement patterns (e.g. anadromous), an important next step is to include a more detailed fish life history into the model and evaluate how the general principles from our strategic model apply. For example, White Sucker (Catostomus commersonii) migrate on a local scale to spawning habitat, and fry and adults have different movement patterns (Geen et al., 1966).

Rarely do disturbances arise in completely pristine river networks these days; most times preexisting disturbances are present. Our work also sheds light on how disturbances interact. We thereby contribute to the research on cumulative effects that is now frequently mandated by government regulations. Upstream movement and downstream transport also alter the patterns of cumulative effects and shift im- 
portance to upstream reaches (see $V I$ ). The findings relate to the well-known SLOSS (single large or several small) debate for conservation planing (Wilcox and Murphy, 1985). Clearly, in river networks, one needs to account for distance according to the network structure rather than geographic distance. Our results for two disturbances indicate that movement patterns and transport of conditions change the optimal conservation design. However, our work assumes that there are no Allee effects, and it is known that population response to fragmentation can change with the shape of density dependence (Dewhirst and Lutscher, 2009).

From a management point of view, our results can be used to determine the least harmful locations for, say, development or risk of toxic contamination in a network. These locations are typically near mouth of the river but they are moved upstream if transport and upstream movement bias are strong. The effects of transportable contaminants on fish populations were studied with a much more detailed tactical model of brown trout (Chaumot et al., 2002, 2003a,b). Their and our models captured only one species and only potadromous movement. One can easily think of scenarios where the downstream location in a highly branched network would be the most vulnerable reach; for example, in an anadromous species (e.g. salmon) where all individuals that return to spawn in a certain watershed have to pass through its mouth. Accordingly, our model should be extended to include multiple species with varying habitat requirements and migration patterns, see for example (Lynch et al., 2011).

More generally, our results relate to questions of managing source-sink populations. A typical result in that literature is that management should focus on highquality patches (sources), but a recent two-patch study found that it may be more helpful to improve the quality of sinks if more individuals are found there (Strasser et al., 2012). When individual movement in a river network is directed, say upstream, then more individuals will be found upstream, and consequently, upstream reaches become the most vulnerable reaches, so that conservation efforts should be focused there. What our study clearly shows, but the two-patch study could not investigate, is the importance of "hubs", i.e. the river mouth central patch in the binary network. While we don't necessarily see a higher density of individuals there, individuals frequently pass through there, giving this patch its importance.

Our modeling framework is a matrix model for a multi-patch population, and as such has a distinct history of successful application in (spatial) demographics (Caswell, 2001; Charles et al., 2009). In particular, sensitivity analysis and simulation tools are well developed, and have recently been extended to age-stage structured populations (Caswell, 2012), which can be interpreted as age-patch models. We were able to assess the importance of each reach in a river network through the 
effect of its quality on the population growth rate. The latter indicator is a robust population endpoint to assess stress in ecology (Forbes and Calow, 2002; Stark and Banks, 2003) and is a projective variable that describes the demographic state of the population (Caswell, 1996); hence it is well suited to answer decision-making questions. Other descriptive population endpoints exist, such as the asymptotic population distribution and the reproductive value. These features were considered by Chaumot et al. (2002, 2003a,b).

Some limitations of our modelling approach should be pointed out. The assumption of a radial tree structure (i.e. homogeneity within levels) is convenient but not necessarily realistic. In particular, dropping this assumption means that the eigenvalue of a randomly generated network cannot necessarily bounded by the linear and the complete binary tree. In fact, it would not even be clear what "the" corresponding lienar an binary tree should be. Another is that we look at the asymptotic growth rate, and therefore consider permanent disturbances to the river network. This approach does not generalize to time-varying disturbances or even stochastic settings. A corresponding fully stochastic metacommunity model was studied by Auerbach and Poff (2011).

One novel aspect in our model is the transport of conditions from one patch to others. Most patch models, either deterministic compartmental models or stochastic metapopulation models, assume that local patch quality is independent of neighboring patch quality. This assumption is clearly not tenable for river networks. Our work strongly suggests that spatial correlations in patch quality have great importance for theoretical population dynamics as well as applied decision-making. We therefore advocate that mathematical tools and quantities - for example indicators of the importance of individual patches to overall population growth rate, viability, and steady-state distribution (Ovaskainen and Hanski, 2003) — be generalized to include spatial correlations induced by river-flow transport or other mechanisms such as ground water.

\section{Acknowledgements}

YS is grateful for an Alexander Graham Bell doctoral scholarship from the Natural Science and Engineering Research Council of Canada (NSERC). FL acknowledges an NSERC discovery grant. Both authors thank Alan Hastings for initial discussions. We also thank Les Stanfield and all the participants for discussions during the 'Trim the Tribs' workshop, held in Ottawa (2012) with financial support from the Toronto and Region Conservation Authority. 


\section{References}

Anderson, K.E., Paul, A., McCauley, E., Jackson, J., and Post, J. (2006). Instream flow needs in streams and rivers: the importance of understanding ecological dynamics. Front. Ecol. Environ., 4(6), 309-318.

Anderson, K.E., Nisbet, R.M., Diehl, S., and Cooper, S. (2005). Scaling population responses to spatial environmental variability in advection dominated systems. Ecol. Lett., 8, 933-943.

Auerbach, D.A. and N.L. Poff (2011). Spatiotemporal controls of simulated metacommunity dynamics in dendritic networks. BioOne 30(1), 235-251.

Bishop, K., Buffam, I., Erlandsson, M., Folster, J., Laudon, H., Seibert, J., and Temnerud, J. (2008). Aqua incognita: the unknown headwaters. Hydrol. Process, $22(8), 1239$.

Caswell, H. (1996). Demography meets ecotoxicology: untangling the population level effects of toxic substances. In M. Newman and C. Jagoe, editors, Ecotoxicology: A hierarchical treatment. Boca Raton, FL (USA): CRC Press, Lewis Publishers. Pages 255-292. CRC Press.

Caswell, H. (2001). Matrix population models. Wiley.

Caswell, H. (2012). Matrix models and sensitivity analysis of populations classified by age and stage: a vec-permutation matrix approach. Theor. Ecol., 5, 403-417.

Charles, S., Bravo de La Parra, R., Mallet, J.-P., Persat, H., and Auger, P. (1998a). A density dependent model describing salmo trutta population dynamics in an arborescent river network. effects of dams and channelling. CR Acad Sci III-Vie, 321(12), 979-990.

Charles, S., Bravo de La Parra, R., Mallet, J.-P., Persat, H., and Auger, P. (1998b). Population dynamics modelling in an hierarchical arborescent river network: an attempt with salmo trutta. Acta biotheor., 46(3), 223-234.

Charles, S., Bravo de La Parra, R., Mallet, J.-P., Persat, H., and Auger, P. (2000). Annual spawning migrations in modelling brown trout population dynamics inside an arborescent river network. Ecol. Model., 133(1), 15-31. 
Charles, S., Billoir, E., Lopes, C., and Chaumot, A. (2009). Matrix population models as relevant modeling tools in ecotoxicology. In J. Devillers, editor, Ecotoxicology Modeling, Emerging topics in Ecotoxicology: Principles, Approaches and Perspectives, volume 2, pages 261-298. Springer.

Chaumot, A., Charles, S., Flammarion, P., Garric, J., and Auger, P. (2002). Using aggregation methods to assess toxicant effects on population dynamics in spatial systems. Ecol. Appl., 12(6), 1771-1784.

Chaumot, A., Charles, S., Flammarion, P., and Auger, P. (2003a). Do migratory or demographic disruptions rule the population impact of pollution in spatial networks? Theor. pop. biol., 64(4), 473-480.

Chaumot, A., Charles, S., Flammarion, P., and Auger, P. (2003b). Ecotoxicology and spatial modeling in population dynamics: An illustration with brown trout. Environ. toxicol. chem., 22(5), 958-969.

Correll, D. (1997). Buffer zones and water quality protection: general principles. In Haycock, N., Burt, T., Goulding, K., Pinay, G. (eds) Buffer zones: their processes and potential in water protection. The proceedings of the international conference on buffer zones, Quest Environmental, pages 7-20.

Courchamp, F., Berec, L., and Gascoinge, J. (2008). Allee effects. Oxford University Press.

Dewhirst, S. and Lutscher, F. (2009). Dispersal in heterogeneous habitats: thresholds, spatial scales and approximate rates of spread. Ecology, 90(5), 1338-1345.

Fagan, W.F. (2002). Connectivity, fragmentation, and extinction risk in dendritic metapopulations. Ecology, 83(12), 3243-3249.

Finn, D.S., Bonada, N., Múrria, C., and Hughes, J.M. (2011). Small but mighty: headwaters are vital to stream network biodiversity at two levels of organization. J. N. Am. Benthol. Soc., 30(4), 963-980.

Forbes, V.E. and Calow, P. (2002). Population growth rate as a basis for ecological risk assessment of toxic chemicals. Phil. Trans. B, 357(1425), 1299-1306.

Geen, G., Northcote, T., Hartman, G., and Lindsey, C.C. (1966). Life histories of two species of catostomid fishes in Sixteenmile Lake, British Columbia, with particular reference to inlet stream spawning. J. Fish. Res. Board Can., 23(11), 1762-1787. 
Goldberg, E.E., Lynch, H.J., Neubert, M.G., and Fagan, W.F. (2010). Effects of branching spatial structure and life history on the asymptotic growth rate of a population. Theor. Ecol., 3(3), 137-152.

Jin, Y., Hilker, F., Steffler, P., and Lewis, M. (2014). Seasonal invasion dynamics in a spatially heterogeneous river with fluctuating flows. Bull. Math. Biol., 76(7), $1522-1565$.

Krasnosel'skii, M.A. (1964). Positive Solutions of Operator Equations. Noordhoff LTD, Groningen.

Krasnosel'skii, M.A. and Zabreiko, P.P. (1984). Geometrical Methods of Nonlinear Analysis. Springer Verlag, Berlin.

Labonne, J., Ravigné, V., Parisi, B., and Gaucherel, C. (2008). Linking dendritic network structures to population demogenetics: the downside of connectivity. Oikos, 117(10), 1479-1490.

Lowe, W. (2006). The trouble with rivers. BioSci., 56(3), 260-263.

Lutscher, F. and Lewis, M.A. (2004). Spatially-explicit matrix models. J. Math. Biol., 48(3), 293-324.

Lutscher, F. and McCauley, E. (2013). A probabilistic framework for nutrient uptake length. Theor. Ecol., 6(1), 71-86.

Lynch, H.J., Grant, E.H.C., Muneepeerakul, R., Rodriguez-Iturbe, I. and Fagan, W.F. (2011). How restructuring river connectivity changes freshwater biodiversity and biogeography. Water Res. Res., 47: W05531.

Meyer, J.L., Strayer, D.L., Wallace, J.B., Eggert, S.L., Helfman, G.S., and Leonard, N.E. (2007). The contribution of headwater streams to biodiversity in river networks. J. Am. Water Res. Assoc., 43(1), 86-103.

Moreno, I. and Billeaud, J. (2015). Wastewater from Colorado Mine Reaches New Mexicos. The Big Story, Associated Press. Published online on 09-August-2015.

Nilsson, C., Ekblad, A., Dynesius, M., Backe, S., Gardfjell, M., Carlberg, B., Hellqviist, S., and Jansson, R. (1994). A comparison of species richness and traits of riparian plants between a main river channel and its tributaries. J. Ecol., 82(2), 281-295. 
Ovaskainen, O. and Hanski, I. (2003). How much does an individual habitat fragment contribute to metapopulation dynamics and persistence? Theor. pop. biol., 64, 481-495.

Padgham, M. and Webb, J.A. (2010). Multiple structural modifications to dendritic ecological networks produce simple responses. Ecol. Model., 221(21), 2537-2545.

Ramirez, J.M. (2012) Population persistence under advection-diffusion in river networks. J. Math. Biol. 65,J 919-942

Samia, Y. (2016) Persistence of River Populations. PhD Thesis, University of Ottawa. http://hdl.handle.net/10393/34495

Samia, Y., Lutscher, F., and Hastings, A. (2015). Connectivity, passability and heterogeneity interact to determine fish population persistence in river networks. J. Roy. Soc. Interface, 12(110), 20150435.

Sarhad, J. and Anderson, K.E. (2015). Modeling population persistence in continuous aquatic networks using metric graphs Fundam. Appl. Limnol., 186, 135-152.

Sarhad, J., and Carlson, R., and Anderson, K.E. (2014). Population persistence in river networks. J. Math. Biol., 69, 401-448.

Stanfield, L., Del Guidice, L., Lutscher, F., Trudeau, M., Alexander, L., Fagan, W., Fertik, R., Mackereth, R., Richardson, J., Shrestha, N., Tetreault, G., and Wipfli, M. (2014). A discussion paper on: Cumulative effects from alteration of headwater drainage features and the loss of ecosystem integrity or river networks. Technical report, Ontario Ministry of Natural Resources.

Stark, J.D. and Banks, J.E. (2003). Population-level effects of pesticides and other toxicants on arthropods. Ann. Rev. Entomology, 48(1), 505-519.

Strasser, C.A., Neubert, M.G., Caswell, H., and Hunter, C.M. (2012). Contributions of high- and low-quality patches to a metapopulation with stochastic disturbance. Theor. Ecol. 5, 167-179.

Thorp, J.H., Thoms, M.C., and Delong, M.D. (2006). The riverine ecosystem synthesis: biocomplexity in river networks across space and time. River Res. Appl., $\mathbf{2 2}(2), 123-147$.

Van Kirk, R.W. and Lewis, M.A. (1997). Integrodifference models for persistence in fragmented habitats. Bull. Math. Biol., 59(1), 107-137. 
Vannote, R.L., Minshall, G.W., Cummins, K.W., Sedell, J.R., and Cushing, C.E. (1980). The river continuum concept. Can. J. Fish. Aquat. Sci., 37, 130-137.

Wilcox, B. and Murphy, D. (1985). Conservation strategy: The effect of fragmentation on extinction. Am. Nat., 125(6), 879-887. 